\title{
CANCER: THE INTERFACE BETWEEN BASIC AND APPLIED RESEARCH
}

\author{
American Association for Cancer Research Conference \\ Baltimore, WA; November 5-8, 1995
}

Bert Vogelstein, Stephen H. Friend, and John A. Minna organized this meeting around a specific question: how do we apply the revolution in the scientific knowledge of cancer to helping patients? Invited speakers presented their strategies, and took the challenge one step further by sharing their "fantasies". At the end of each presentation, the feasibility of their ideas was put to a vote: "Would you give the speaker a million dollars to do the research?", adding a taste of reality. This innovative format stimulated much more discussion and questioning than is typical for meetings of similar size (about 400 attendants). What follows is a small sampling of the many studies discussed.

Highlighting the importance of basic research, Scott Hawley (University of California, Davis) described how studies in a lower organism can provide insights into human cancer. His group cloned the mei-41 gene in Drosophila melanogaster and found it to be the structural and functional homolog of the human ATM gene which is mutated in the cancer susceptibility syndrome ataxia telangiectasia. By screening in $D$. melanogaster for mutations that enhance or suppress mei-4l mutations, two genes that interact with mei-4l were isolated. Hawley's fantasy is to use the fly to fish out a set of human genes that interact with ATM, thereby elucidating the precise biochemical pathway leading to cancer development.

A number of other pathways involved in the pathogenesis of cancer have been identified in recent years by describing the genetic alterations found in human tumor samples. The key players in these pathways also represent potential molecular targets for the design of cancer drugs. With this in mind, Michael H. Wigler (Cold

Address correspondence to: Laura P. Bonetta, The Picower Institute Press, 350

Community Drive, Manhasset, NY 11030.
Spring Harbor Laboratory, New York) developed RDA (representational difference analysis), a technique based on subtractive hybridization to identify probes from chromosomal regions undergoing deletions or amplifications in tumor cells. Jeffrey Sklar (Brigham and Women's Hospital, Boston) described a technique to microdissect particular chromosomal fragments, for example those showing a loss of heterozygosity in tumor samples, and to amplify complementary DNA (cDNA) clones hybridizing to these fragments. The isolated cDNAs can then be tested for point mutations or other relevant structural alterations by comparison to cDNAs derived from normal tissues. His fantasy was to eventually map all mutations in human cancer.

Jeffrey M. Trent (National Center for Human Genome Research, Bethesda) used a similar microdissection-mediated cDNA capture technique to isolate genes from chromosomal regions that are amplified in human cancer. His fantasy was to use these cDNAs to generate a high capacity system to monitor the expression of multiple genes and to ultimately provide customized therapy for cancer patients. The approach he proposed consisted of preparing differently colored fluorescent probes from either normal or tumor cells which would be hybridized to microarrays of cDNA clones. This would then allow the colorcoded detection of differences in patterns of gene expression, serving as an in vivo sensor for clinical diagnosis. With a similar goal in mind, Kenneth W. Kinzler (Johns Hopkins School of Medicine, Baltimore) has developed a technique for the serial analysis of gene expression (SAGE), which allows the quantitative and simultaneous analysis of a large number of transcripts. The technique is being applied to describing expression patterns at different stages of tumor development using colorectal cancer as a model system. Kinzler's fantasy was to be able to correlate 
a particular expression pattern with a clinical prognosis and to apply this to customizing chemotherapy for patients.

Given this exciting basic research, the next question is how is it being translated into therapy? Allen Oliff (Merck Research Laboratories, West Point, PA) has developed highly selective inhibitors of farnesyl-protein transferase (FPTase), the enzyme responsible for the farnesylation of the Ras oncoprotein, a protein mutated in $25 \%$ of all human cancers. Transgenic mice harboring a mutant Ras gene in their germ line develop breast and salivary carcinomas; administration of FTPase inhibitors resulted in complete regression of tumors in this model system. Furthermore, no evidence of toxicity was found in any of the mice after treatment with the drug for up to 3 months. Dr. Oliff predicted that FTPase inhibitors will go to clinical studies within the next year.

p53 is the gene mutated in the majority of human cancers. A.J. Levine (Princeton University), in collaboration with Dr. Barry Bloom (Albert Einstein School of Medicine, New York), has used BCG (an attenuated strain of the tuberculosis agent) containing p53 to immunize mice. The animals were shown to produce cytotoxic $\mathrm{T}$ lymphocytes (CTLs) which recognize and kill tumor cells with mutations in the p53 gene, but did not recognize normal cells. This is thought to be due to the fact that mutant $\mathrm{p} 53$ protein is usually expressed at much higher levels and has a longer half-life than its wild-type counterpart, thereby allowing it to be "seen" by the immune system. Levine's fantasy was to develop a vaccine against human cancers containing p53 mutations.

Immunization of cancer patients was also the fantasy of Steven A. Rosenberg (Surgery Branch, NCI, Bethesda). Tumor infiltrating lymphocytes (TIL) recognize cancer antigens on murine and human cancers. In clinical trials, tumor nodules have been removed from patients with melanoma and grown in vitro with TILs and IL-2. When the
TILs were re-introduced into the patients, $37 \%$ of them underwent regression. Rosenberg's group has now isolated and characterized the melanoma antigens gp 100, MART-1, and TRP-1. His fantasy is to generate TILs with great tumor antigen reactivity by sensitizing cells in vitro to specific peptides. In order to improve immunogenicity, modified peptides could be rationally designed.

Finally, one approach deemed to hold promise for a cancer cure is gene therapy. Michael Blaese (NCI, Bethesda) described the delivery of "suicide" genes specifically to cancer cells. A clinical trial of patients with high-grade glioblastomas or metastatic cancer to the brain was started at the Clinical Center of the NIH. 19 lesions in 15 patients were treated by local injections of murine fibroblasts producing retroviral vectors carrying the thymidine kinase (tk) gene followed, 7 days later, with the intravenous administration of ganciclovir for 14 days. Expression of the tk gene produced an enzyme which converts ganciclovir into a highly toxic agent, capable of killing the tk-containing cells and their neighbors (bystander effect). Antitumor activity was demonstrated in about one half of patients. Dr. Blaese's fantasy is to develop a gene therapy vector that replicates exclusively in tumor cells. One potential approach is to delete the El region of adenovirus so that the virus can no longer replicate in normal cells with functional p53. This viral vector might, however, replicate in tumor cells lacking p53 function.

Considering the many widely differing approaches discussed at this stimulating meeting, it was not surprising that when presentations were put to a vote, scientists in the audience differed widely in their opinions and preferences. Maybe in a few years another meeting should be organized to see who got the million dollars and, more importantly, if it was well spent. 